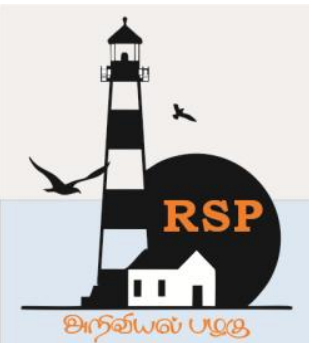

INTERNATIONAL RESEARCH JOURNAL ON ADVANCED SCIENCE HUB

RSP SCIENCE HUB

(The Hub of Research Ideas)

Available online at www.rspsciencehub.com

\title{
3D Printing Innovation during Covid-19 Pandemic
}

Siddavatam rammohan reddy ${ }^{1}$,Balaji krushna potnuru ${ }^{2}$

${ }^{1,2}$ Assistant Professor, Department of Mechanical Engineering, Malla Reddy Engineering College

(Autonomous), Maisammaguda, Rangareddy (Dist.), Secunderabad, Telangana -500100, India.

sriramreddy.276@gmail.com ${ }^{1}$

\begin{abstract}
$3 D$ Printers are playing a huge role in helping to fight against COVID-19.3D Printing also known as additive manufacturing, refers to processes used to create a three-dimensional object in which layers of material are formed under computer control to create an object. With $3 D$ printing innovation these days, we can easily transform any design into a 3D model. The utilization of $3 D$ Printers in medical fields are growing quickly and are required to reform human services. The corona virus infection-19 has spread all over the world and stances diverse challenges to healthcare offerings. Health care staff rely on Personal Protective Equipment to protect themselves and their patients from infection. Hospitals around the globe are running out of PPE for treating COVID Patients. The constrained accessibility of PPE has impacted many countries ability to react to the emergency. The $3 D$ printing network is ready to overcome this international scarcity. The target of the current work is to examine the $3 D$ printing innovation with regards to a Covid-19 pandemic and to analyze which kind of PPE can be printed utilizing 3D Printers. The beneficial 3D prints for Covid-19 are Face shield mask, Safety goggles, nasal swabs, Ventilators, Oxygen Venturi valves, hands-free door openers, Tweezers, Handy tool for buttons, Respirator mask, 3D Printing pill dispenser, medical devices and surgical tools and so on.
\end{abstract}

Keywords: 3D Printing, PPE and COVID-19

\section{Introduction}

Corona virus infection (COVID-19) is a newly discovered disease and it is an irresistible disease. The infection especially spread via droplets produced by an infected person coughs, sneezes or respires. Those droplets are too heavy to hold the air and rapidly fall on floors or surfaces. When you are close to someone who has corona virus or contacts a contaminated surface then your eyes, nose or mouth, you will become infected by breathing in the virus. Each patient/individual requires a separate mask and other personal protective equipment (PPE). How all of the sudden hospital could increase ventilators, protective equipment and some other spare parts needed. These segments can be printed effectively with Additive manufacturing process/3D Printers. Additive manufacturing (AM) refers to a process by which the material deposited using digital 3D modelling data to build up a product in layers. Combination of these layers forms a solid object. Progressively, the term 3D Printing is used as a synonym for additive manufacturing.[1-4]

\section{3D Printing Technologies}

In the age of COVID-19 supply chain got dispirited. 3D Printing is a supply chain substitute in this crucial period of scarcity. 3DP innovations will play a significant part in supporting hospital workers efforts in the midst of this emergency. 3D printing is an AM process in which material is solidified under machine control to construct 3D/Solid objects from a computerized file. 3D Printing gives the designer the ability to quickly 
convert ideas into 3D models or prototypes. The various 3D printing techniques are shown in Fig.1

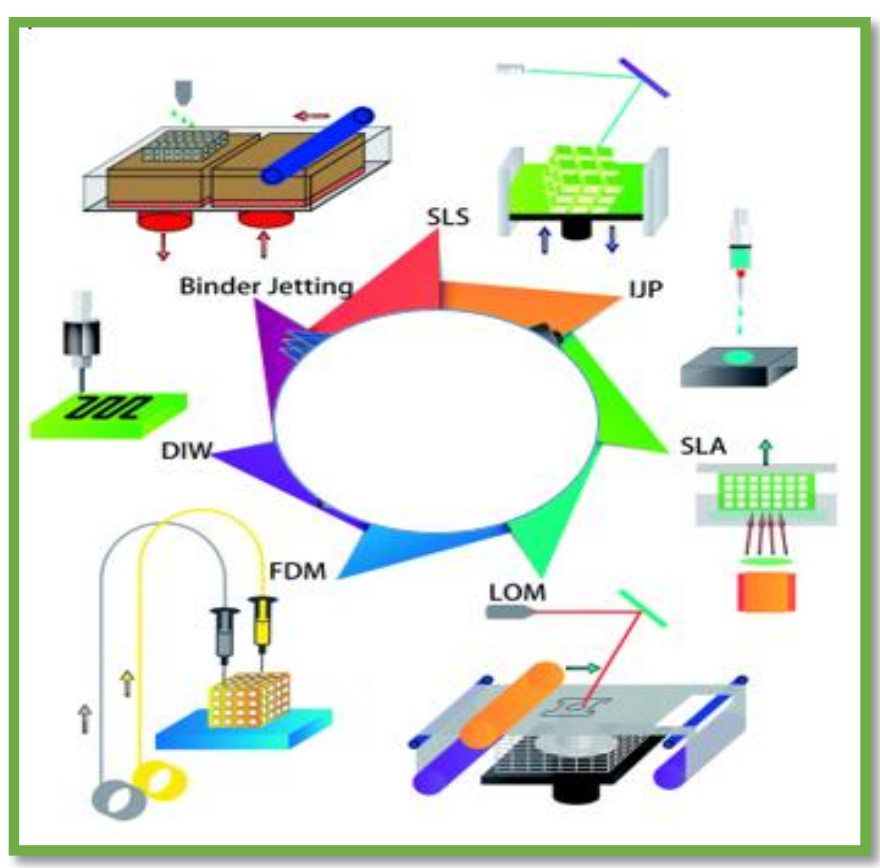

Fig.1 3D Printing Techniques

\subsection{Fused Deposition Modeling (FDM)}

FDM printers are most popular in modernday to print Personal protective equipment's and contributing about $60 \%$ in the overall production of PPE. FDM is layer AM process that uses thermoplastic material to produce prototypes and 3D models. the commonly used materials are PLA (polylactic acid) \& ABS (acrylonitrile butadiene styrene) widely used to print the component. FDM Printer consist of a print head, build platform and raw material in the form of a filament as shown in Fig.2

The FDM method starts by slicing CAD data into layers. The information is then moved to a machine which develops the part layer by layer on the build platform then fed the spool of thermoplastic and support materials are used to make each cross-section of the part. The material slowly extruded through a dual heated nozzle. The extrusion nozzle is preciously laid-down both support material and thermoplastic material on the proceeding layers.[5-9] this procedure is repetitive layer by layer based on exact design coordinates. As soon as it is exposed to a cooler environment. the molten material becomes solid. The extrusion nozzle continues to move in a horizontal $\mathrm{X} \& \mathrm{Y}$ plane while the build platform moves down building the part layer by layer. 3D printer accuracy depends upon the minimum distance the nozzle can travel vertically (Z-axis). the finished part is taken out from the build platform and the supporting material is removed to achieve the desired shape of the model. FDM is utilized in aerospace, automotive, industrial, commercial and Medical field.

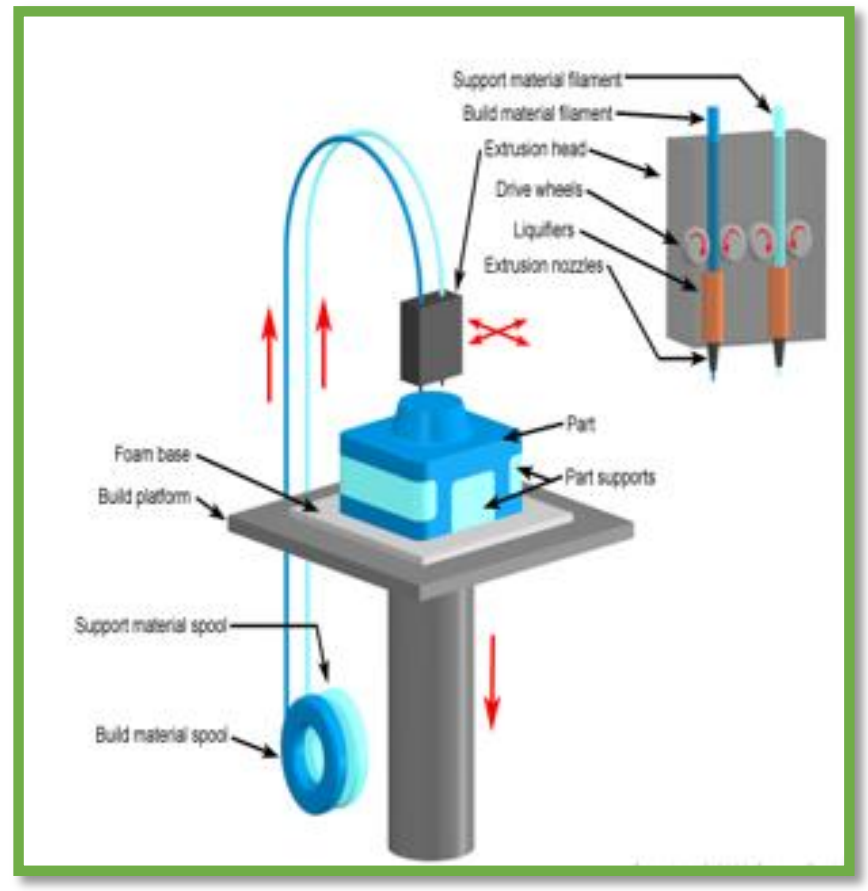

Fig.2 FDM Process

\section{3D Printing Process}

The following are the steps to be followed in the 3D Printing process.

Step1: First 3D Model of the object is created by using CAD Software's like PTC Creo, Autodesk Fusion360, CATIA, Solid works etc. Another way of creating the $3 \mathrm{D}$ digital file is by using a $3 \mathrm{D}$ object scanner.

Step2: We need to convert it into .STL or .OBJ format once we have finished the CAD model.

Step3: There are numerous 3D slicing software's are accessible. They are CURA, Slic3r, idea Maker, Z-Suite, MakerBot Print etc. after the file is sliced, it generates tool path in form of G-Code, which is the code that a $3 \mathrm{D}$ printer recognizes and follows.

Step4:The printer movements are saved/ stored in $\mathrm{SD}$ card or sent to the printer over wireless or 
www.rspsciencehub.com

cable depending on the printer.

Step5: The parameters for printing are set for the printer and filament is fed to the print head.

Step6: 3D Printer builds the model on a platform layer by layer by depositing the material.

Step7: Part and its supports are removed from the build platform.

Step8: Finally, we need to clean, polish the part and paint component if required.

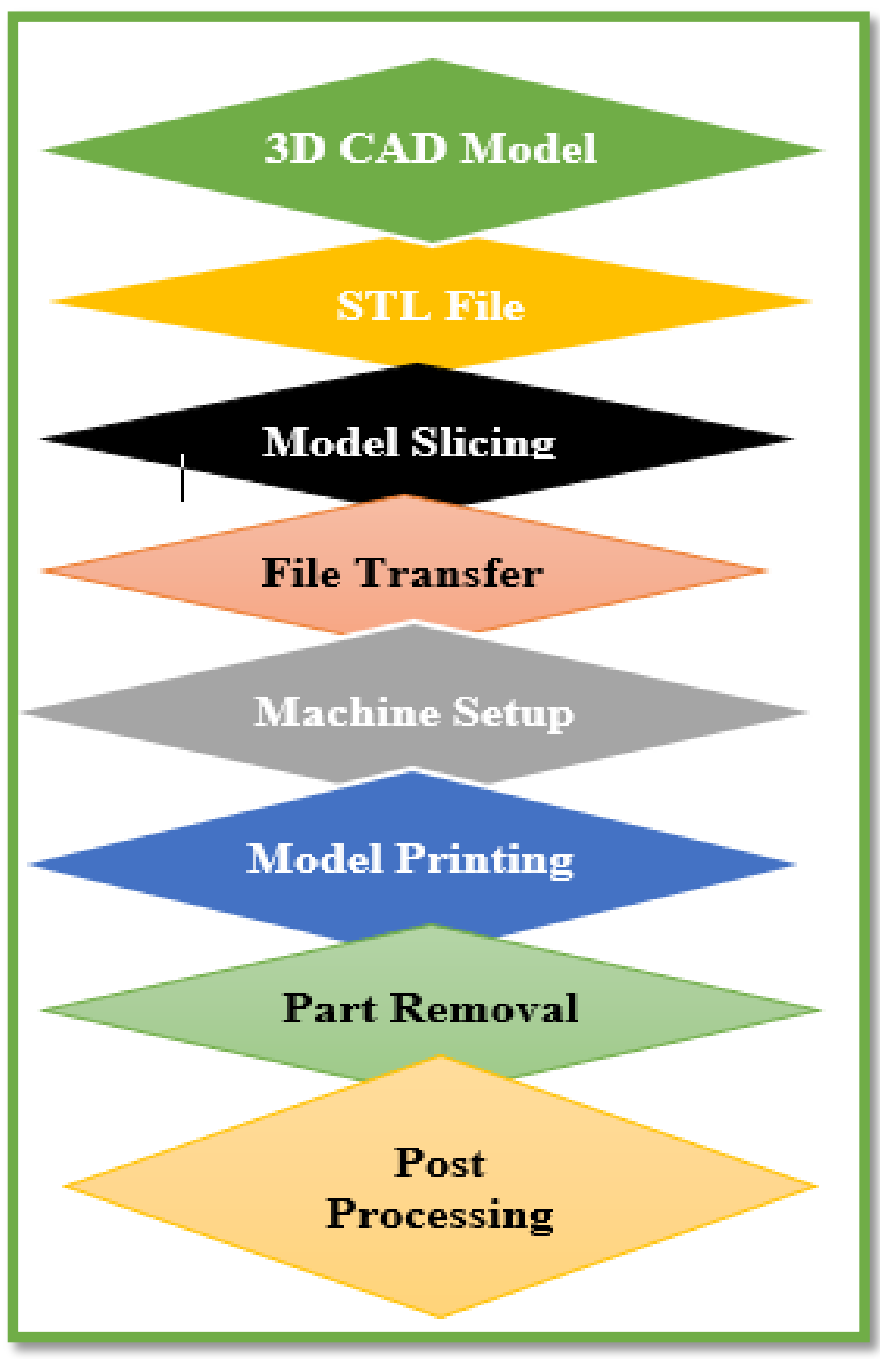

Fig. 3: Steps in 3D printing Process

\section{3D Printable PPE and Parts of Medical equipment}

\section{1 face shield:}

During the Coronavirus pandemic, the face shield has become an important part of the PPE utilised by health care workers. the face shields that cover the full face. There are many types of face shields, but most medical workers are looking
Volume 02 Issue 08 August 2020

for is a piece of clear, curved plastic suspended in front of the wearer's face by a headband and it should be comfortable and fit appropriately. Hospitals, universities, and research centres have investigated the ways to produce the best face shield. The various kinds of face shield as shown in Fig.4
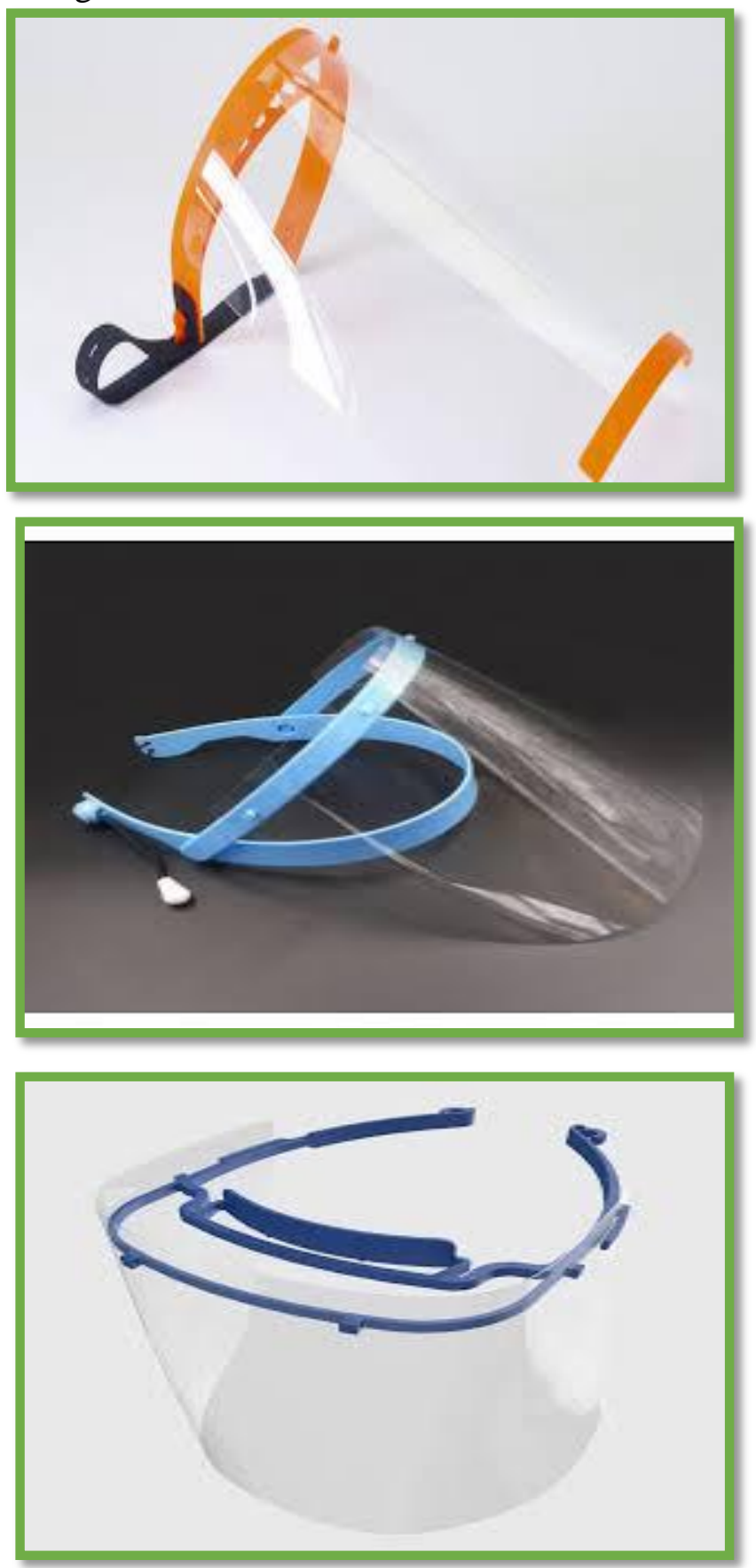

Fig.4 Different Types Face Shield

We have designed face shield mask frame in PTC Creo and we printed it in HYDRA-200 printing machine. The printed face shield mask frame as shown in Fig.5 

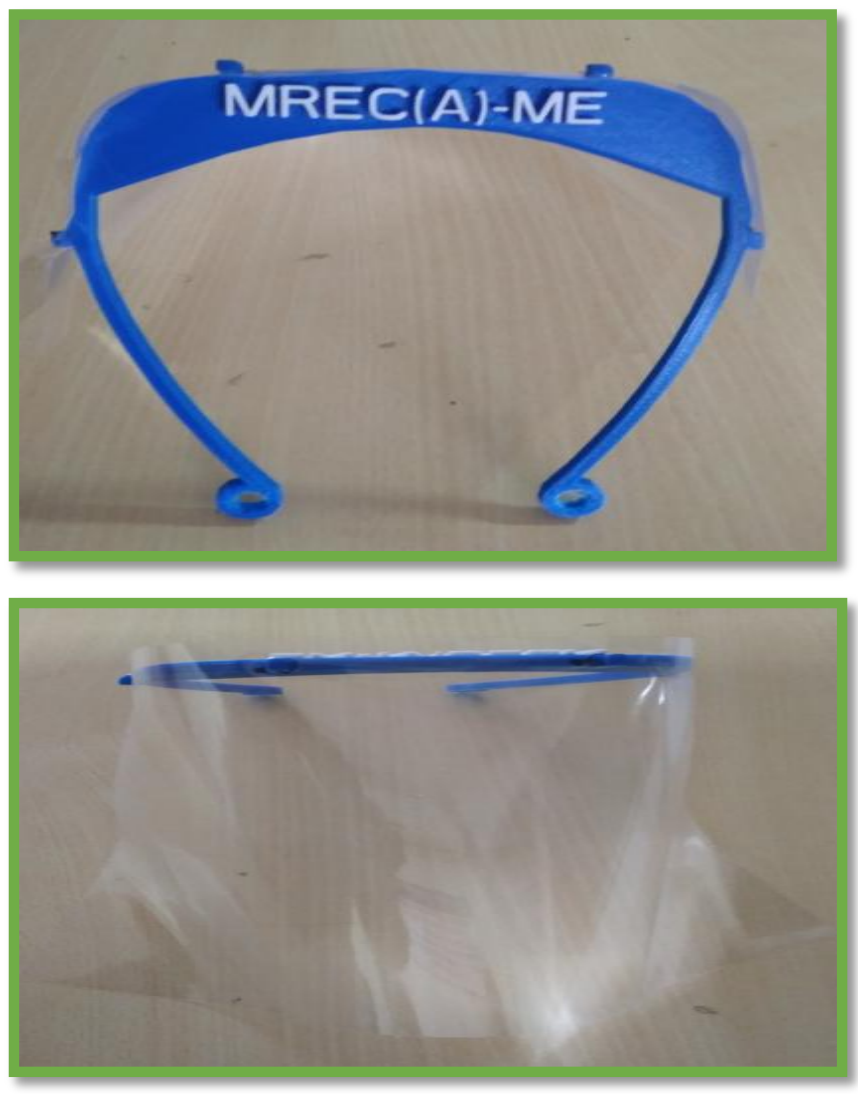

Fig.5 Face shield frame printed with our 3D

\subsection{Protective Goggles} printer

The safety goggles are made from PLA and come with transparent cover protection. the nose region is comfortably shaped and the length of the ear clips can be adjustable. Safety goggles are shown in Fig.6
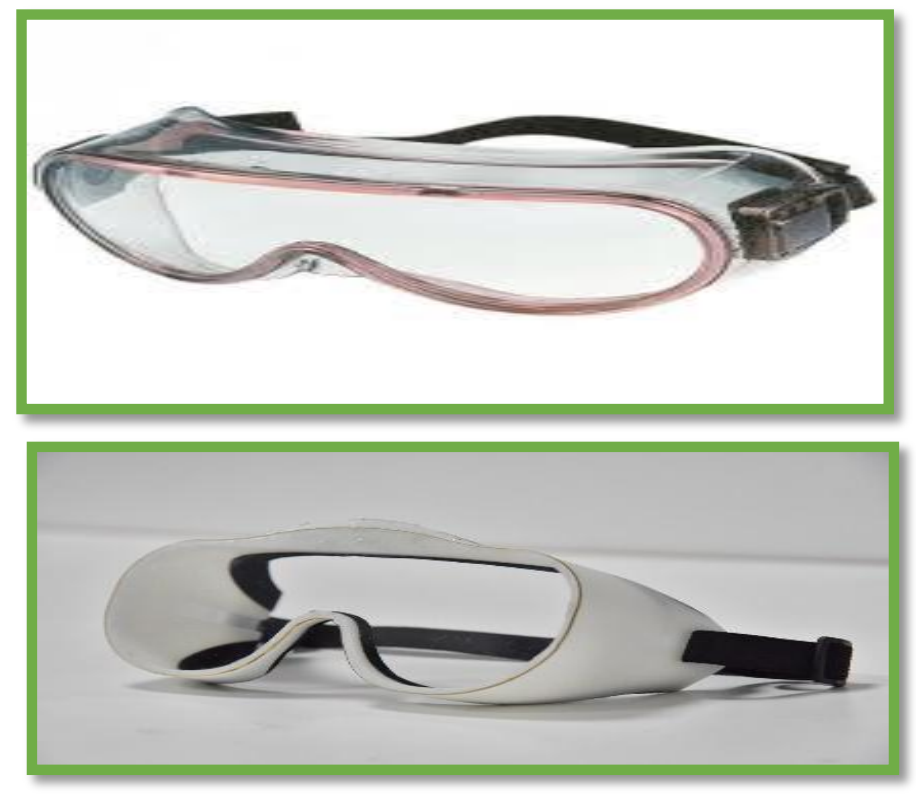

Fig.6 Safety Goggles

\subsection{Respiratory Valves}

Coronavirus patients require breathing machines have recently run out of the respiratory valves expected to interface the patients to the machine. The initial supplier couldn't meet the demand and the medical clinic immediately ended up in an emergency. Rapidly responding to the situation 3d Printers are utilized to meet hospital requirements. 3D printed respiratory valves shown in Fig.7
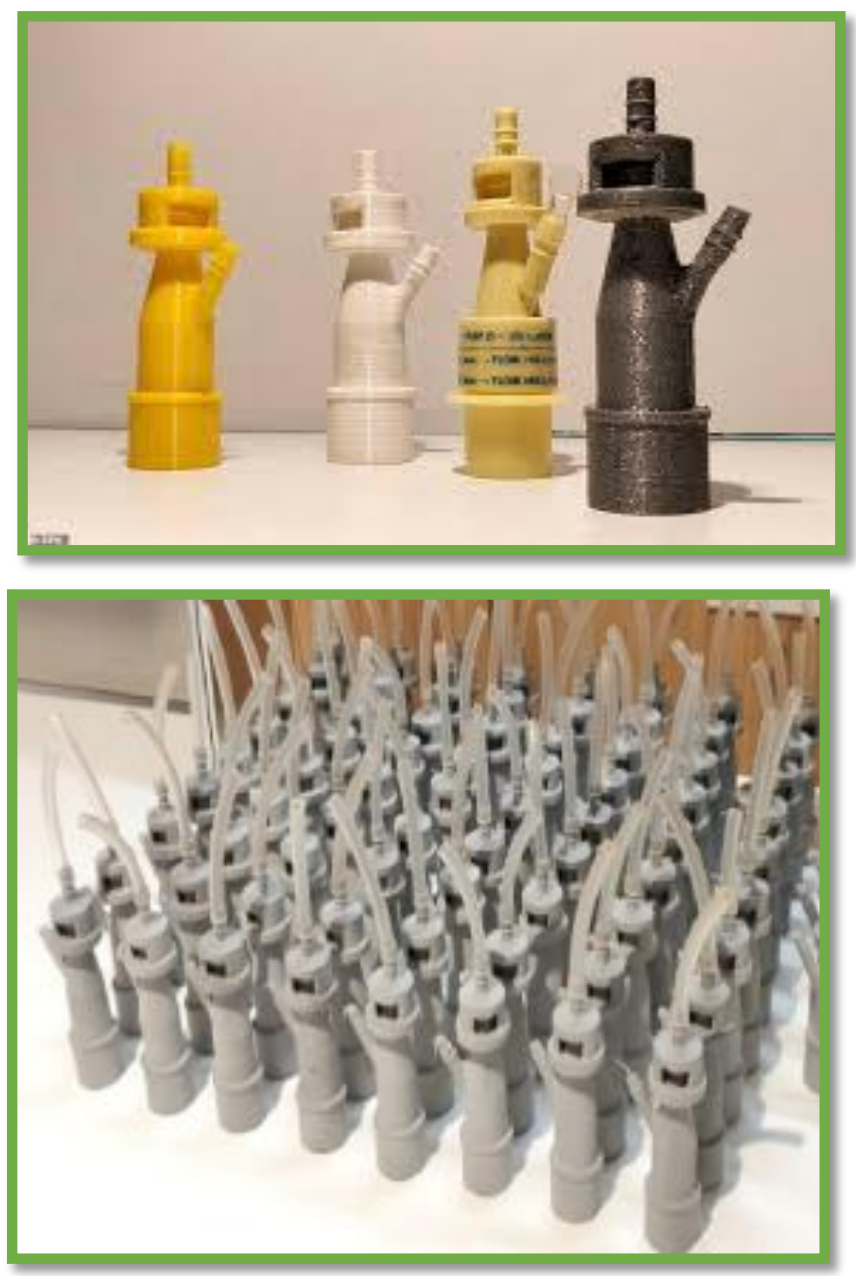

Fig. 7 Respiratory Valves

\subsection{Hands-free door handle attachment}

Door handles, specifically in public areas such as offices and hospitals are subject to a lot of physical contacts. That turns into a hotspot for virus spreading. Door handle requires screws and nuts to fix it. Hands-free door attachment handle as shown in Fig.8 

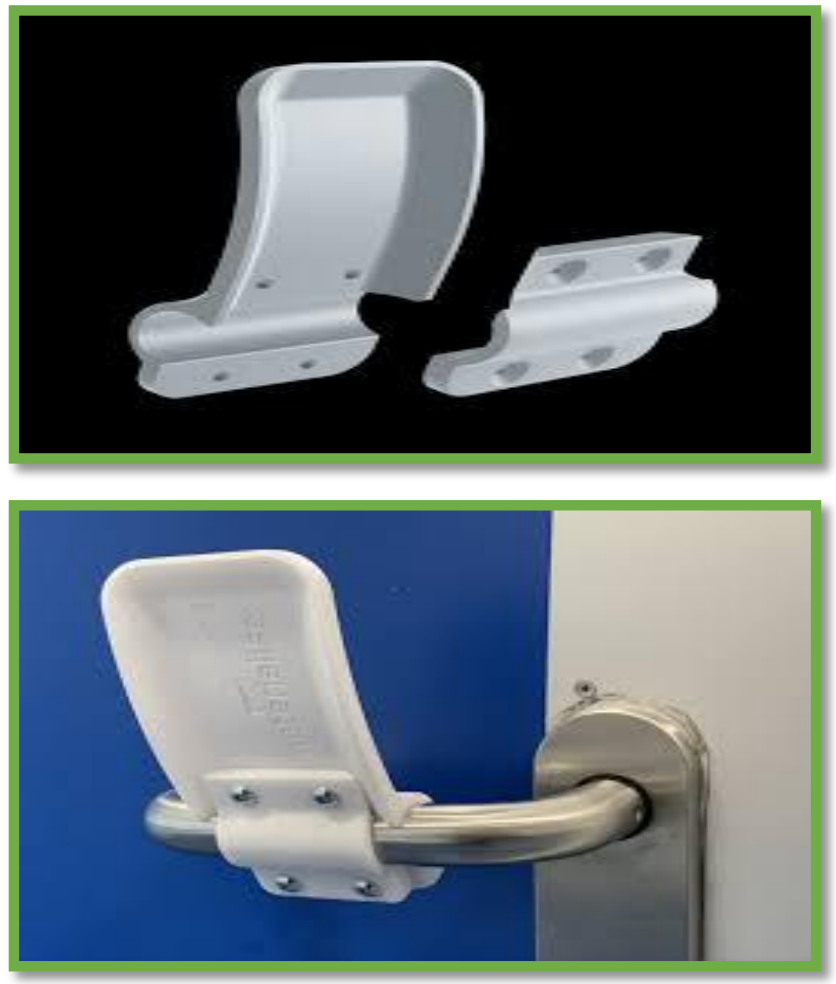

Fig.8 Hands-free door handle attachment

\subsection{D Printed Respirator Face Mask}

3D Printing technology is permitting general society to step up to help the deficiency of PPE. Safety and effectiveness remain the way of fighting the COVID-19 Pandemic. This mask protects the nose and mouth from germs and it is more remarkable than throwaway surgical masks. A soft and flexible material is twig to the upper edge of the mask. 3D printed face mask with reusable a border as shown in Fig.9
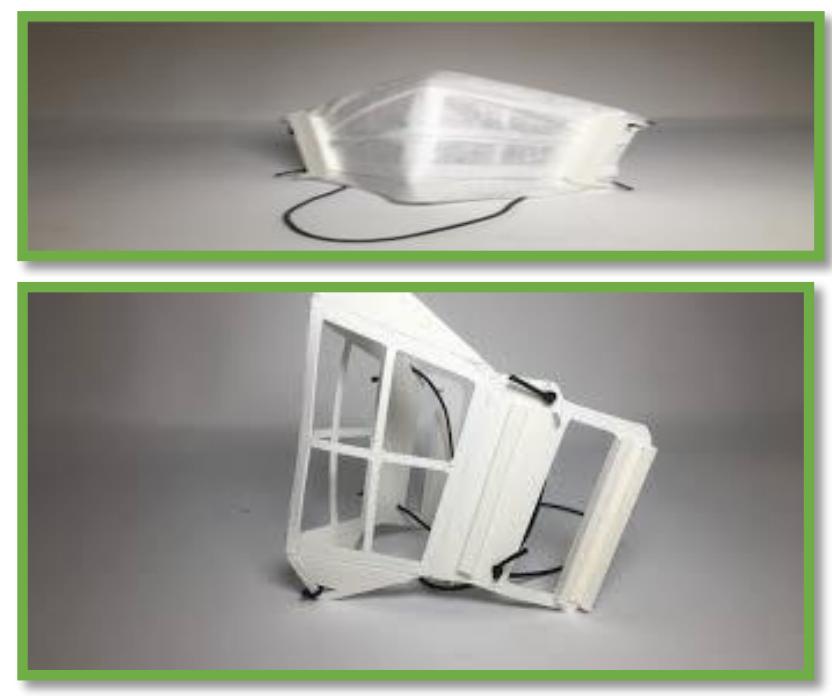

Volume 02 Issue 08 August 2020

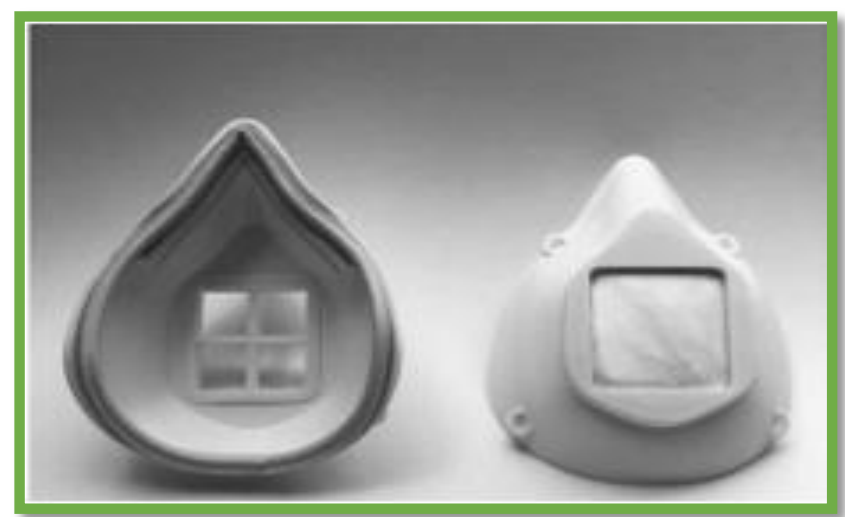

Fig. 9 3D printed face mask

\subsection{Handy tool}

The objective of the handy tool is to avoid direct contact with handles, buttons, bags and other daily objects outside of our home. You can use 3d Printed handy tool to pull or push an entryway handle on every day, open the car door, push a button and it can help you to press ATM buttons. Handy devices are helpful for people to communicate with daily tasks without touching or getting excessively near items across open spaces. The various 3D printed hand tools are shown in Fig.10
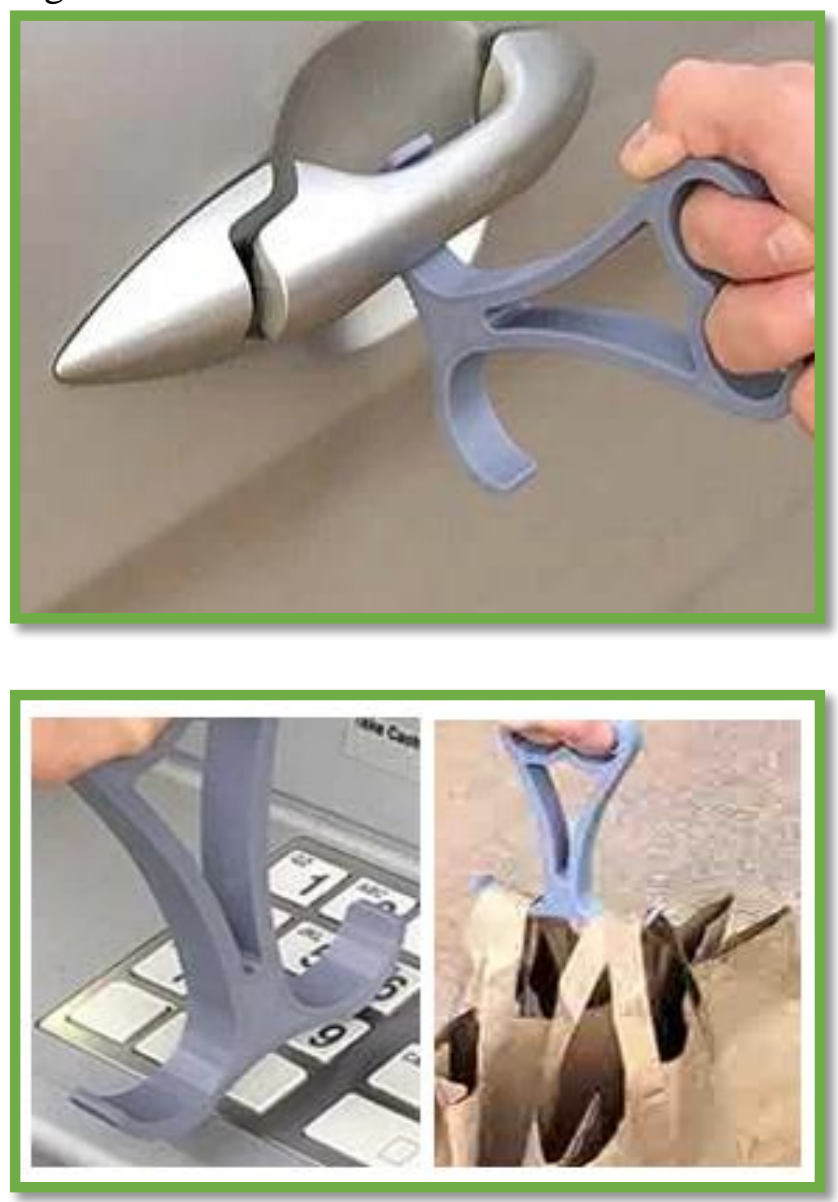


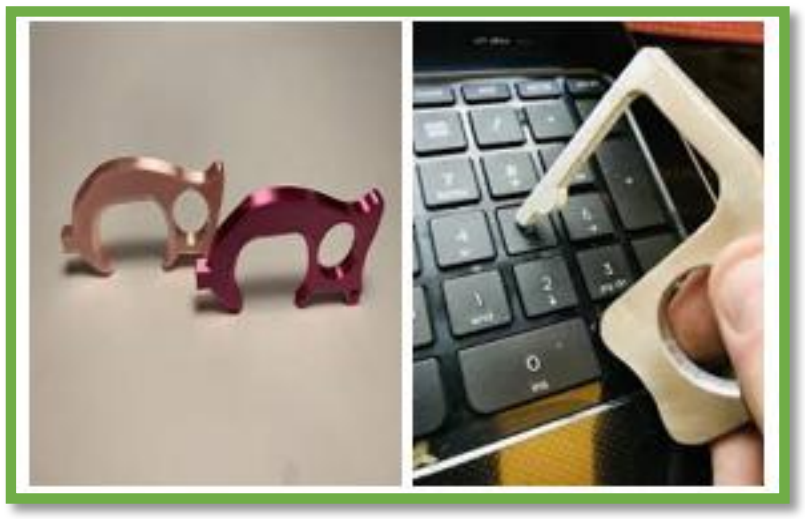

Fig.10 Handy Tools

\section{Conclusion}

The main utilization of the 3DP advancements for battling against COVID-19 infection have been summed up. the threedimensional printing (3DP) innovations are offering endless benefits, flexibilities and customizations. 3D Printing became an essential method to print personal protective equipment's just as lessening an opportunity to showcase and improve product quality. 3D Printing innovation could reform and reshape the world. Advances in 3D printing technology will radically change and improve the way we produce the PPE equipment to meet the drastically rising globalization. 3D printing brings unlimited possibilities of designs in product development. In the future, organs and body parts might be Printed utilizing 3D Printing techniques.

\section{References}

[1].Nick Vordos, Despina A. Gkika, George Maliaris, Konstantinos E. Tilkeridis, Anastasia Antoniou, DimitriosV. Bandekas, Athanasios Ch.Mitropoulos, "How 3Dprinting and social media tackles the PPE shortage during Covid-19 pandemic" Journal of Safety scienceElsevier June 2020.

[2].SatyamChaturvedi, AmartyaGupta, VimalKrishnanS, Anil K. Bhat "Design, usage and review of a cost effective and innovative face shield in a tertiary care teaching hospital during COVID-19 pandemic" Journal of orthopaedic- Elsevier May 2020.

[3]. SunpreetSingh, ChanderPrakash, Seeram
Ramakrishna 'Three-dimensional printing in the fight against novel virus COVID-19: Technology helping society during an infectious disease pandemic" Technology in Society-Elsevier Aug 2020.

[4].Siddavatam Rammohan Reddy "A Lithophane Model Making Process To 3d Printers" International Journal of Mechanical Engineering and Technology (IJMET), Volume 11, Issue 5, 2020

[5]. Vinod G. Gokhare, Dr. D. N. Raut, Dr. D. K. Shinde "A Review paper on 3D-Printing Aspects and Various Processes Used in the 3D-Printing"-, (IJERT) Vol. 6 Issue 06, June - 2017

[6]. Peng Chang, Hui Mei, Shixiang Zhou, Konstantinos G. Dassios and Laifei Cheng "3D printed electrochemical energy storage devices" Journal of medical chemistry-Jan 2019

[7].Engineering Product Design "https://engineeringproductdesign.com/add itive-manufacturing-process-steps/"

[8]. 3D printing Industry "https://3dprintingindustry.com/news/3dprinting-community-responds-to-covid-19-andcoronavirus-resources-169143/"

[9]. Design boom

"https://www.designboom.com/design/handy-opensource-tool-slow-the-spread-of-covid-19-04-222020/ 